\title{
Intervenção em educação e saúde sobre obesidade infantojuvenil em uma unidade escolar de Belém do Pará
}

\author{
Intervention in education and health on childhood juvenile obesity in a school unit in Belém \\ do Pará
Intervención en educación y salud sobre la obesidad juvenil de la infancia en una unidad escolar en Belém do Pará

Richard Franco da Silva Moraes ${ }^{1 *}$, Raquel Eloi Guedes ${ }^{1}$, Nathalia Lais Lima Rocha ${ }^{1}$, Bianca Abdelnor Hanna Piqueira Diniz ${ }^{1}$, Gabriela Freitas da Silva Ferreira ${ }^{1}$, Gabriella Sousa de Oliveira ${ }^{1}$, Ana Catarina da Paz Holodniak¹, Karen Lury Abe Emotoํㅜ, Gabriela Ladeia da Silva ${ }^{1}$, Osvaldo da Silva Peixoto'.

\section{RESUMO}

Objetivo: Promover intervenção contra a obesidade por meio de palestra sobre educação em saúde para estudantes em uma escola de um município do Pará e avaliar o estado nutricional dos estudantes. Métodos: Tratase de um estudo clínico, quantitativo e longitudinal com uma amostra de 100 alunos de 11 a 16 anos. Resultados: Das 100 crianças, $17 \%$ estão com sobrepeso/obesidade, $55 \%$ estão eutróficas e $28 \%$ estão abaixo do normal para idade. Após a ampla análise de dados foi montado uma estratégia para palestra que abordasse todos os problemas evidenciados, foi elaborado um folder explicativo que nos auxiliou durante a explanação e as crianças se mostraram bastante interessadas e participativas sendo que grande parte delas se demonstraram motivados a mudarem algumas condutas do cotidiano. Conclusão: Observou-se discreta prevalência de sobrepeso e obesidade dos estudantes. A intervenção foi satisfatória abrangendo o máximo de participantes possível que se demonstraram interessadas e aprenderam sobre a temática, o que se infere que as informações sobre obesidade infanto-juvenil deveriam sempre ser abordadas nas escolas.

Palavras-chave: Obesidade, Educação em saúde, Infantojuvenil.

\section{ABSTRACT}

Objective: Promote intervention against obesity through a lecture on health education for students at a school in a municipality in Pará and assess the nutritional status of students. Methods: This is a clinical, quantitative, and longitudinal study with a sample of 100 students aged 11 to 16 years. Results: Of the 100 children, $17 \%$ are overweight / obese, $55 \%$ are eutrophic and $28 \%$ are below normal for age. After extensive data analysis, a lecture strategy was developed to address all the problems highlighted, an explanatory folder was elaborated that helped us during the explanation and the children were very interested and participatory, and most of them were motivated to change some of them. everyday behaviors. Conclusion: There was a slight prevalence of overweight and obesity among students. The intervention was satisfactory, covering as many participants as possible who showed interest and learned about the theme, which implies that information on childhood and youth obesity should always be addressed in schools.

Keywords: Obesity, Health education, Juvenile children.

\section{RESUMEN}

Objetivo: Promover la intervención contra la obesidad a través de una conferencia sobre educación sanitaria para estudiantes de una escuela en un municipio de Pará y evaluar el estado nutricional de los estudiantes. Métodos: Estudio clínico, cuantitativo y longitudinal con una muestra de 100 estudiantes de 11 a 16 años. Resultados: De los 100 niños, $17 \%$ tienen sobrepeso / obesidad, $55 \%$ son eutróficos y $28 \%$ están por debajo de lo normal para la edad. Después de un extenso análisis de datos, se desarrolló una estrategia de conferencia para abordar todos los problemas resaltados, se elaboró una carpeta explicativa que nos ayudó durante la explicación y los niños estuvieron muy interesados y participativos, la mayoría de ellos motivados para cambiar algunos de ellos. comportamientos cotidianos. Conclusión: Hubo una ligera prevalencia de sobrepeso y obesidad entre los estudiantes. La intervención fue satisfactoria, cubriendo la mayor cantidad posible de participantes que mostraron interés y aprendieron sobre el tema, lo que implica que la información sobre la obesidad infantil y juvenil siempre debe abordarse en las escuelas.

Palabras clave: Obesidad, Educación sanitaria, Niños menores.

${ }^{1}$ Centro Universitário Metropolitano da Amazônia (UNIFAMAZ), Belém - PA. *E-mail: richard-franco@hotmail.com SUBMETIDO EM: 4/2020 


\section{INTRODUÇÃO}

A obesidade é uma doença crônica caracterizada pelo excesso de gordura corporal, que causa prejuízos à saúde do indivíduo. É o agravo nutricional que mais preocupa este início do século XXI, tanto pelo incremento rápido e progressivo na sua prevalência quanto pelo fato de o fenômeno ocorrer em todas as fases da vida, desde crianças a idosos. Destaca-se, sobremaneira, a situação em crianças e adolescentes, visto que os agravos nessa faixa etária perduram por toda a vida (BRASIL, 2016).

No Brasil, segundo dados do Ministério da Saúde de 2014, estima-se que 20\% das crianças sejam obesas, e a região norte, mais precisamente na cidade de Belém, no Estado do Pará, 50,4\% da população infantojuvenil estão acima do peso e $16,1 \%$ estão obesos. Segundo o Estudo do Instituto Brasileiro de Geografia e Estatística (IBGE), em média por ano, cada belenense consome 5.694 quilos de gordura.

A elevada quantidade de calorias por grama, comum à maioria dos alimentos ultra processados, é um dos principais mecanismos, de acordo com a publicação, que desregulam o balanço de energia e aumentam o risco de obesidade. Associados ao tamanho das porções e à frequência da ingestão, mesmo alimentos regionais como tapioca, tucumã, farinha de mandioca e o açaí, também contribuem para o aumento do sobrepeso dos Belenenses (OMS, 2016).

Segundo a Organização Mundial De Saúde (OMS), em 2014, 48\% das crianças menores de cinco anos e que estavam acima do peso ou obesas, viviam na Ásia e $25 \%$ na África. Os números no continente africano quase dobraram desde 2000, de 5,4 milhões para 10,3 milhões. Dados nacionais dos anos de 2010 e 2014 revelaram que uma em cada três crianças de cinco a nove anos estava com sobrepeso ou obesidade. Entre os adolescentes, $21,7 \%$ do sexo masculino e $19,4 \%$ do sexo feminino apresentaram esse problema. Esses resultados reafirmaram o sobrepeso como o principal desvio nutricional nessa faixa etária (OMS, 2014).

A crescente prevalência de obesidade infantil está associada ao surgimento de comorbidades anteriormente consideradas como doenças "adultas", incluindo diabetes mellitus tipo 2, hipertensão, doença hepática gordurosa não alcoólica, apneia obstrutiva do sono e dislipidemia. A causa mais comum de obesidade em crianças é um balanço energético positivo devido à ingestão calórica em excesso do gasto calórico combinado com uma predisposição genética para ganho de peso (SEEMA K e AARON SK, 2017).

As crianças e adolescentes estão subordinados às condições socioeconômicas e culturais das suas famílias, o que influencia a prática de atividade física, a quantidade e o tipo de alimentos disponíveis. Somado a isso, a atual geração de crianças e adolescentes tem livre acesso às tecnologias como computadores, videogames, celulares e tablets, sem limite do tempo de permanência em frente das telas, sendo que acesso favorece ao maior tempo investido em atividades que exigem menor gasto energético (AMA O, et al., 2013)

Os maus hábitos das crianças, como má alimentação, inatividade física pode influenciar o desenvolvimento de doenças crônicas já citadas, sendo o contexto social e econômico em que a pessoa está inserida determinante, isso porque os entornos físicos e padrões sociais dificultam a compra de alimentos nutritivos, uma vez que o consumo alimentar de crianças brasileiras é marcado por frequências elevadas de inadequação no consumo de micronutrientes, sobretudo ferro, vitamina $A$ e zinco. Essas inadequações não se apresentam apenas sob o aspecto da deficiência, mas também por meio de excessos, como observado para o consumo energético (AMA O, et al., 2013).

Portanto, cenários como esses, derivam-se, os fatores que predispõem toda a família ao excesso de peso, devido à contribuição do ambiente obesogênico. Além disso, a obesidade é cada vez mais impulsionada pela globalização e urbanização, a exposição a ambientes insalubres que aumenta em países de alta, média e baixa renda e em todos os grupos socioeconômicos. Também a comercialização de alimentos pouco saudáveis e bebidas não alcoólicas são identificadas, como um fator importante para o aumento do número de crianças com sobrepeso e obesidade, particularmente no mundo em desenvolvimento, gerando uma serie de distúrbios físicos, biológicos, psicológicos e sociais nas crianças do século (MINISTÉRIO DO PLANEJAMENTO, 2010).

A Insatisfação com a imagem corporal e bullying também são desfechos que afetam a saúde de crianças e adolescentes em idade escolar. Na obesidade infantil estão presentes os fatores psicossociais e, dentre as 
dimensões presentes nesses fatores, está a imagem corporal. Há dois tipos de distúrbios com a imagem corporal: o perceptual, que se relaciona com a subestimação do tamanho do corpo; e o atitudinal, que envolve a insatisfação com a forma ou o tamanho do corpo, onde as crenças dos indivíduos a respeito dos seus corpos podem acarretar em satisfação ou insatisfação com a imagem corporal ( SOUZA ECG, et al., 2012; PALLAN MJ, et al., 2011)

Além da imagem corporal, o bullying também ocasiona um impacto psicológico negativo, onde as crianças têm percepções e visões de suas experiências e podem resistir em relatar experiências constrangedoras ou estressantes, como ocorre com as vítimas de bullying. Um estudo realizado nas 26 capitais brasileiras e no Distrito Federal encontrou que $5,4 \%$ dos avaliados relataram ter sofrido bullying quase sempre ou sempre nos últimos 30 dias (SCHNAIDER SK, et al., 2012; MALTA DC, et al., 2014).

Crianças pequenas que vivem em famílias historicamente marginalizadas correm o risco de se tornarem adolescentes com obesidade e, posteriormente, adultos com aumento de morbidades relacionadas à obesidade (ORDWAY MR, et al., 2018; CUNHA LF, 2014; SILVA MC, et al., 2013)

É possível inferir que a obesidade infantojuvenil, infelizmente está associada com consequências psicológicas e sociais negativas, principalmente no que diz respeito as experiências escolares com o bullying. O estudante pode sofrer descriminação de diversas maneiras, decorrentes de agressões físicas e/ou verbais que repercutem em exclusão social, traumas que podem ser permanentes, como ansiedade, depressão, baixa autoestima e problemas comportamentais, comprometendo o rendimento escolar, bem como a qualidade de vida desses estudantes. Há também comportamentos ligados aos distúrbios alimentares como, a bulimia e anorexia; o consumo de álcool, drogas, laxantes e até mesmo medicamentos para emagrecer que agravam o estado de saúde desses jovens (MALTA DC, et al., 2014).

Os contextos socioeconômicos nos quais os jovens estão inseridos também devem ser levados em consideração, uma vez que as condições culturais das crianças e adolescentes envolvidas são moldadas perante os familiares e pessoas de convívio próximo, influenciando a prática de atividade física, a quantidade e o tipo de alimento disponível. O entorno social pode, por vezes, dificultar o acesso da criança e do adolescente a alimentos saudáveis, gerando práticas inadequadas e cíclicas que contribuem para o excesso de peso. É de fundamental importância compreender tal realidade, visto que a obesidade é etiologicamente definida como uma condição multifatorial (MARIZ LS, et al., 2015; HERNANDES F E VALENTINI MP, 2010).

Portanto, por ser o local onde as crianças fazem uma das suas principais refeições diárias, o espaço mais indicado aos alunos para a prática de atividade física, e ser uma das principais localidades onde o jovem estabelece suas relações sociais, o trabalho de prevenção contra a obesidade é necessário e pertinente na escola. Assim, quando posta em prática, a ação em educação nutricional, aliada à promoção da atividade física, oferece ao jovem uma perspectiva de crescimento saudável, resultando em uma melhor qualidade de vida, bem como um prognóstico favorável que objetiva reduzir as complicações tardias derivadas de um padrão nutricional inadequado, além de uma melhor relação social com seu entorno (ALVES JGB, 2013; CARVALHO CA, 2015).

O primeiro passo para ajudar um jovem a ter um peso saudável é conversar. Podemos definir metas saudáveis para perda de peso e ajuda com monitoramento e suporte. Planos de perda de peso para crianças se concentram em hábitos de vida saudáveis. Um estilo de vida saudável é bom para todos, mesmo que a perda de peso não seja o objetivo principal (KUMAR S, et al., 2017; GROSSMAN DC, et al., 2017; GOVERNMENT HM, 2016; ALCÂNTARA AOR, et al., 2013).

Devido a elevada incidência de obesidade infantil na Capital Paraense, prevenir a obesidade infantil significa diminuir, de uma forma racional, a incidência de doenças crônico-degenerativas. Ao ser notado um elevado índice de obesidade na unidade escolar, no município de Belém pertencente a área delimitada da Unidade Saúde da Família, o presente trabalho, almeja promover a ação de intervenção em educação nutricional, aliada à promoção da atividade física, na tentativa de estimular hábitos mais saudáveis na população infantojuvenil escolar, reduzindo os agravos e complicações das doenças crônicas causadas pela obesidade através de palestra educativa. 


\section{MÉTODOS}

Trata-se de um estudo clínico, quantitativo e longitudinal. O estudo foi realizado na Escola Estadual de Ensino Fundamental e Médio, no bairro da Pedreira, no município de Belém, Estado do Pará. A população do estudo consistiu em estudantes devidamente matriculados na Escola Estadual supracitada e que possuem perfil de sobrepeso e obesidade na faixa etária de 11 a 17 anos. A amostra consistirá em aproximadamente 100 alunos.

Participaram todos os estudantes com perfil de sobrepeso e obesidade segundo tabela do Índice de Massa Corpórea (IMC) determinada pela sociedade brasileira de pediatria. Participaram todos aqueles que apesar de não estarem com sobrepeso ou obesos, manifestem interesse em participar das palestras, no qual os responsáveis estejam de acordo e assinado o Termo de Consentimento Livre e Esclarecido e os menores assinando o Termo de Assentimento.

Não participaram crianças e/ou adolescentes juntamente com os seus responsáveis que não tiveram interesse em participar da pesquisa, além dos estudantes não matriculados na Escola Estadual de Ensino Fundamental e Médio e crianças não compreendidas na faixa etária de 11 a 17 anos.

Este estudo realizado preconizou os documentos Internacionais e a Res. 466/12 do Ministério da Saúde, e teve início após a submissão e aprovação do projeto pela Plataforma Brasil, sob número do Certificado de Apresentação para Apreciação Ética (CAAE) - 75635417.2.0000.5701, número do parecer 2.398.282. Da Autorização da instituição de ensino, Termo de Consentimento de Livre e Esclarecido (TCLE) assinado pelos responsáveis e Termo de Assentimento assinado pelos alunos.

O instrumento de coleta utilizado foi uma ficha de avaliação direcionada para o estado nutricional, criado pelos autores da pesquisa para facilitar a identificação dos participantes e coleta dos dados em que continham perguntas sobre classe de alimentos mais consumidos nos lanches na escola e em casa, quantas vezes na semana ingeriam doces e refrigerantes, quantidade de vezes que se alimentavam no dia, se realizavam atividade física fora da escola, se tinham o habito de comer frutas e verduras, se tinha pai e/ou mãe com hipertensão ou diabetes mellitus tipo 2 e se já sofreram algum tipo de bullying relacionado ao peso.

Todas as crianças e adolescentes que participaram do projeto passaram por coleta de medidas antropométricas como peso e altura utilizando materiais certificados pelo INMETRO. Foram realizados encontros educativos com palestras sobre obesidade, mostrando para os participantes como isso afetariam suas vidas atualmente e no futuro. Foram abordados também métodos a serem adotados que previne a obesidade e auxilia na perda de peso como: alimentação saudável, prática de atividades físicas e esportivas.

A análise dos dados foi realizada por metodologia quantitativa, sendo que os mesmos foram digitados em um banco de dados para execução da análise estatística. $O$ banco de dados, bem como as tabelas e os gráficos foram construídos no programa Bioestat 5.0, Excel e WHO AnthroPlus.

\section{RESULTADOS}

A amostra foi constituída de 100 crianças sendo 51 crianças do sexo masculino, com idade variando entre 11 e 14 anos e média de 12.43 anos (IC95\% 12.10 - 12.76) e por 49 crianças do sexo feminino (Figura 1), com idade entre 11 e 16 anos, com média de 11.77 anos (IC95\% $11.44-12.10$ ). A idade média das 100 crianças foi igual a 12.11 (IC95\% 11.87 - 12.34).

Como mostrado pelos índices podemos observar que 17\% das crianças estão com sobrepeso/obesidade e $55 \%$ estão eutróficos (Gráfico 1) entrando no grupo da palestra sobre como adquirir hábitos mais saudáveis. Por outro lado, observamos uma quantidade considerável de crianças abaixo do IMC esperado para idade (28\%).

Isso provavelmente se dá pela deficiência quali-quantitativa dos alimentos consumidos no dia a dia. A palestra por sua vez, por ter tido viés de orientação de hábitos de vida e alimentares bem como contribuiu para adequação nutricional desse grupo de crianças. 
Figura 1 - Comparação das curvas de crescimento das crianças incluídas no estudo com a curva de referência da OMS (2007), segundo o índice IMC/idade, geral (A) e por sexo (B).
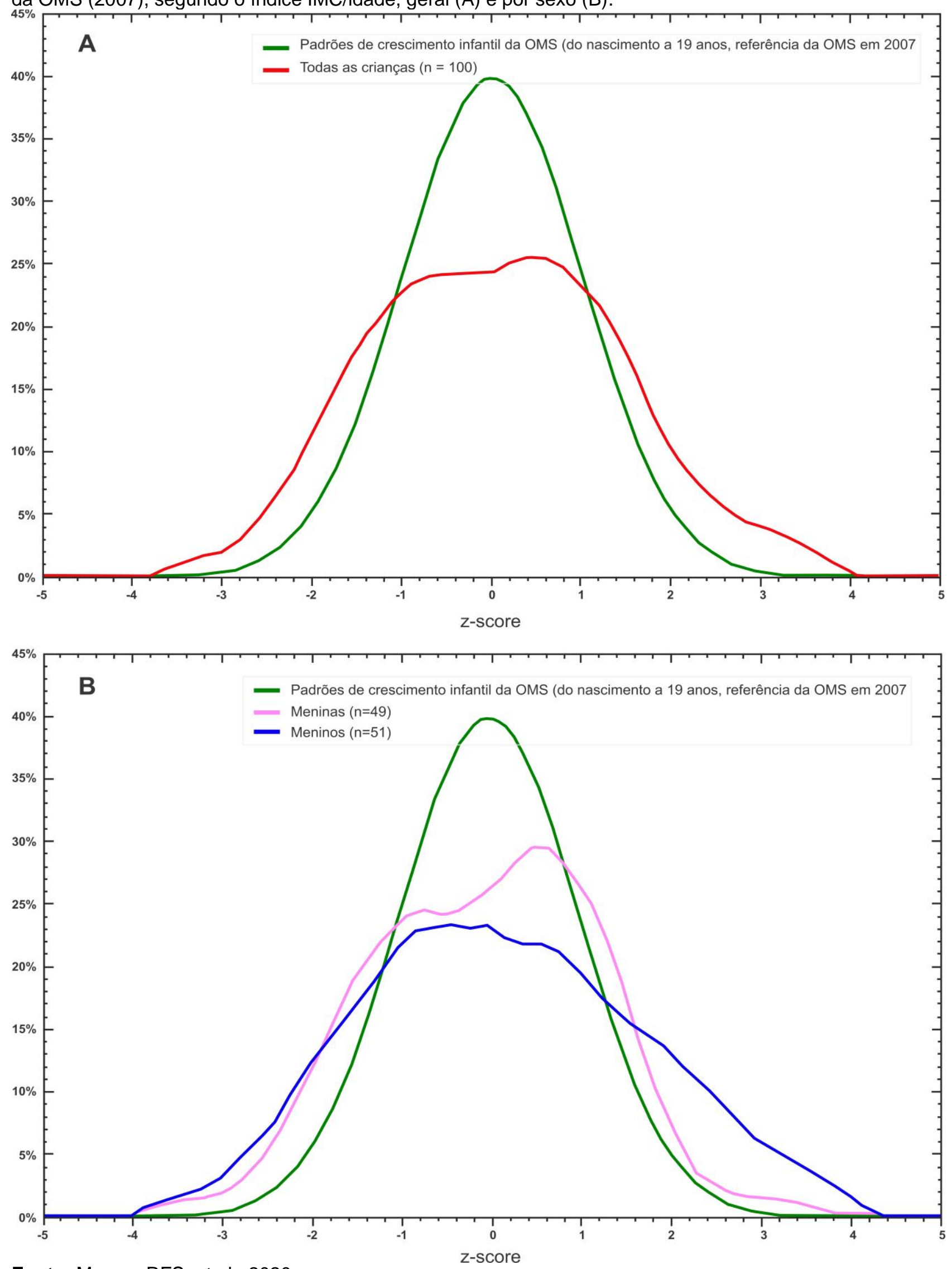

Fonte: Moraes RFS, et al., 2020. 
Gráfico 1 - Estado nutricional das crianças incluídas no estudo, segundo idade e sexo, pelo índice Avaliação IMC segundo OMS.

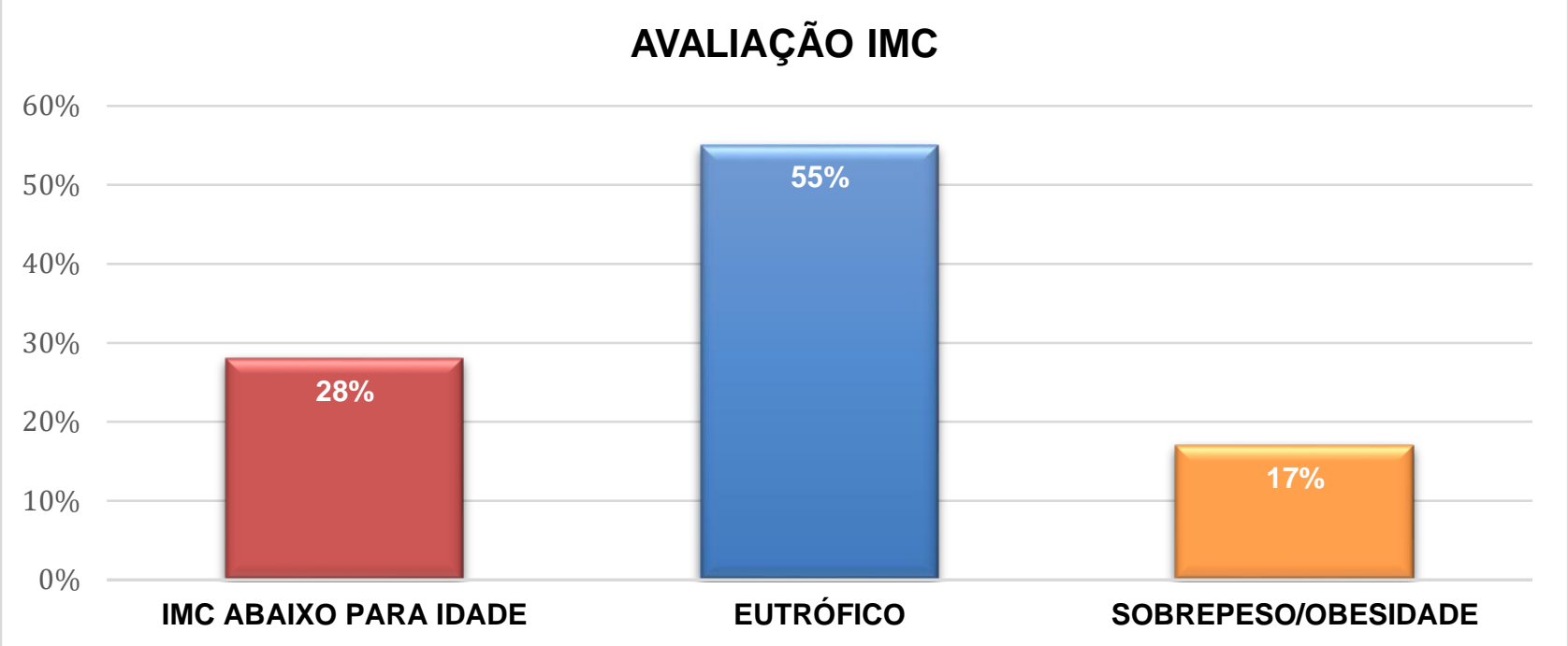

Fonte: Moraes RFS, et al., 2020.

Entre as faixas etárias, a prevalência de crianças com peso acima do considerado adequado segundo os padrões da OMS variou de $0 \%$ a $100 \%$, não havendo, no entanto, diferenças significativas entre os grupos ( $p$ $=0.2720$ ), assim como não se observou associação em relação à classificação nutricional, pelo IMC/idade, com os sexos $(p=0.3680)$ (Tabela 1$)$.

Tabela 1 - Estado nutricional das crianças incluídas no estudo, segundo idade e sexo, pelo índice IMC/ldade, 2018.

\begin{tabular}{|c|c|c|c|c|c|c|c|c|}
\hline \multirow{3}{*}{ Variável } & \multirow{3}{*}{$\mathbf{N}$} & \multicolumn{6}{|c|}{ Diagnóstico nutricional } & \multirow{3}{*}{ p-valor } \\
\hline & & \multicolumn{2}{|c|}{$\begin{array}{c}\text { Baixo IMC para } \\
\text { idade }<-2\end{array}$} & \multicolumn{2}{|c|}{$\begin{array}{l}\text { Eutrófico } \\
\geq-2 a<+1\end{array}$} & \multicolumn{2}{|c|}{$\begin{array}{c}\text { Sobrepeso/Obesidade } \\
\geq+1\end{array}$} & \\
\hline & & $\mathbf{n}$ & $\%$ & $\mathbf{n}$ & $\%$ & $\mathbf{n}$ & $\%$ & \\
\hline \multicolumn{9}{|l|}{ Idade (anos) } \\
\hline 11 & 41 & 14 & 34,1 & 19 & 46,3 & 8 & 19,6 & \multirow{6}{*}{0.2720} \\
\hline 12 & 29 & 3 & 10,3 & 20 & 69 & 6 & 20,7 & \\
\hline 13 & 11 & 5 & 45,5 & 6 & 54,5 & 0 & 0 & \\
\hline 14 & 17 & 6 & 35,3 & 9 & 53 & 2 & 11,7 & \\
\hline 15 & 1 & 0 & 0 & 0 & 0 & 1 & 100 & \\
\hline 16 & 1 & 0 & 0 & 1 & 100 & 0 & 0 & \\
\hline \multicolumn{9}{|l|}{ Sexo } \\
\hline Masculino & 51 & 15 & 29,4 & 25 & 49 & 11 & 21,6 & \multirow{2}{*}{0.3680} \\
\hline Feminino & 49 & 13 & 26,6 & 30 & 61,2 & 6 & 10,2 & \\
\hline
\end{tabular}

Legenda: ${ }^{\star} T$ este $\mathrm{G}$ de independência. $\neq$ Valores expressos em Escores $\mathrm{z}$.

Fonte: Moraes RFS, et al., 2020.

Como fatores de risco observados a partir de questionário respondido pelos alunos, observamos o consumo exagerado de doces e refrigerantes bem como uma alimentação rica em carboidratos, frituras e guloseimas (Gráfico 2). Associado a isso, mais da metade das crianças (55\%), não praticam nenhuma atividade física fora da escola. Em fatores para doenças hereditárias associadas à obesidade como Hipertensão Arterial Sistêmica (HAS) e Diabetes Mellitus tipo 2 (DM2), 27\% tinha pai e/ou mãe com HAS ou DM2. Um dado positivo é que $82 \%$ das crianças possuem o hábito de comer frutas e verduras.

Ao serem questionados sobre a quantidade de refeições diárias podemos observar os 2 extremos, crianças que se alimentam abaixo do recomendado, $<3$ vezes ao dia (16\%) e crianças que se alimentam acima do recomendado, $>6$ vezes ao dia (9\%), fatores que contribuem tanto para sobrepeso e obesidade quanto para desnutrição (Gráfico 2). Além disso cerca de $80 \%$ das crianças com sobrepeso ou obesidade revelaram que já sofreram algum tipo de bullying relacionado ao seu peso. 
Gráfico 2 - Hábitos alimentares das crianças da unidade escolar em \%.

\section{QUANTIDADE DE VEZES QUE SE ALIMENTAM NO DIA}

\section{QUANTIDADE DE VEZES POR SEMANA QUE INGEREM REFRIGERANTES E DOCES}

\section{TIPOS DE ALIMENTOS \\ PREDOMINANTES NOS LANCHES}

100

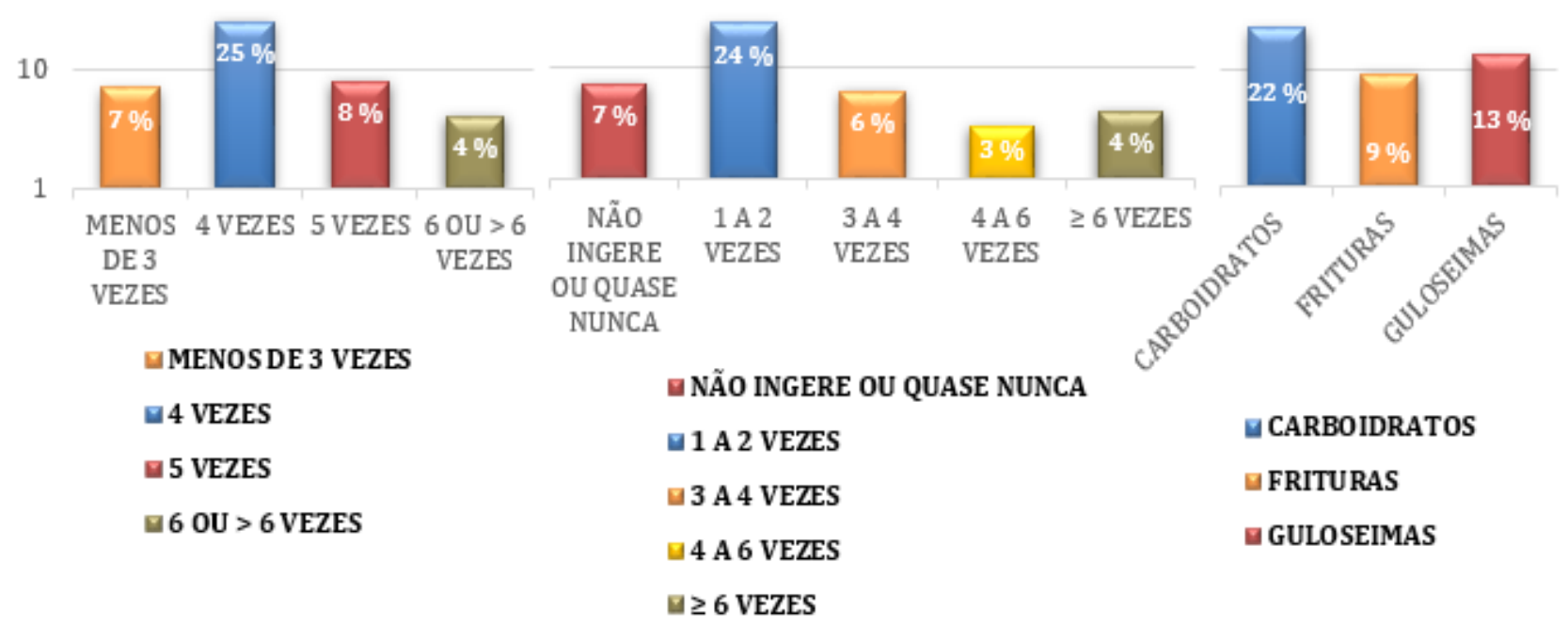

Fonte: Moraes RFS, et al., 2020.

Após a ampla análise de dados, foi elaborada uma estratégia para palestra que abordasse todos os problemas evidenciados. Foi elaborado um folder explicativo com linguagem de fácil entendimento e ilustrativo que nos auxiliou durante a explanação das informações. Inicialmente, houve resistência de parte da turma em assimilar os ensinamentos referentes a nutrição e qualidade dos alimentos.

No decorrer da intervenção, após apresentação de exemplos práticos que foram facilmente associados ao cotidiano, as crianças se mostraram bastante interessadas e participativas, realizaram perguntas que envolviam a temática além de tirarem dúvidas após o término da atividade. De modo geral, grande parte do grupo demonstrou motivação para mudarem algumas condutas rotineiras, como levar o lanche de casa e a procurar alguma atividade física fora da escola como andar de bicicleta, jogar futebol, fazer natação e dança.

\section{DISCUSSÃO}

Ao correlacionar todos os dados analisados para construção desta pesquisa, com os resultados encontrados para levantamento de dados e posteriormente à realização da intervenção, foi notória a grande variedade de fatores que levaram a necessidade de realização desse estudo.

Entende-se que o local onde as crianças fazem uma das suas principais refeições diárias, o espaço mais indicado aos alunos para a prática de atividade física, e ser uma das principais localidades onde o jovem estabelece suas relações sociais, o trabalho de prevenção contra a obesidade é necessário e pertinente na escola, uma vez que estes jovens passam em média 6 horas diárias nesse ambiente.

Com a intervenção pode-se criar uma perspectiva de crescimento saudável, resultando em uma melhor qualidade de vida, bem como um prognóstico favorável que objetiva reduzir as complicações tardias derivadas de um padrão nutricional inadequado, além de uma melhor relação social com seu entorno (ALVES JGB, 2013; CARVALHO CA, 2015).

Durante a intervenção, os pesquisadores questionaram aos participantes sobre hábitos de vida e alimentares praticados dentro e fora do ambiente escolar o que foi determinante para delimitar os principais assuntos a serem abordados durante a palestra de intervenção. Uma vez que a causa mais comum de obesidade em crianças é um balanço energético positivo devido à ingestão calórica em excesso do gasto calórico combinado com uma predisposição genética para ganho de peso (SEEMA K e AARON SK, 2017). 
Durante a coleta de dados foram obtidas mais afirmativas em relação ao bullying, já que eram notados comentários depreciativos enquanto eram aferidas as medidas antropométricas das crianças mostrando que bullying também ocasiona um impacto psicológico negativo, onde as crianças têm percepções e visões de suas experiências e podem resistir em relatar experiências constrangedoras ou estressantes (SCHNAIDER SK, et al., 2012; MALTA DC, et al., 2014).

As crianças e adolescentes estão subordinados às condições socioeconômicas e culturais das suas famílias, o que influencia a prática de atividade física, a quantidade e o tipo de alimentos disponíveis (AMA O, et al., 2013). Isso demonstrado no Gráfico 2 em que as qualidades dos alimentos são de baixo valor comercial o que facilita o acesso por parte das crianças de baixa renda.

No entanto, por serem alimentos com baixo teor nutritivo além de causar predisposição à obesidade leva também à desnutrição como visto em $28 \%$ dos participantes. Os estudo demonstram que crianças que vivem em famílias historicamente marginalizadas correm o risco de se tornarem adolescentes com obesidade e, posteriormente, adultos com aumento de morbidades relacionadas à obesidade (ORDWAY MR, et al., 2018; CUNHA LF, 2014; SILVA MC, et al., 2013).

Tendo-se como base que o primeiro passo para estimular uma vida saudável em crianças e adolescentes é a conversa, montamos toda uma linha de suporte para que todos se sentissem contemplados durante a intervenção, já que, um estilo de vida saudável é bom para todos, mesmo que a perda de peso não seja o objetivo principal (KUMAR S, et al., 2017; GROSSMAN DC, et al., 2017; GOVERNMENT HM, 2016; ALCÂNTARA AOR, et al., 2013).

Nota-se também uma discreta proporção na frequência em relação ao gênero, na literatura, entre os adolescentes, $21,7 \%$ do sexo masculino e $19,4 \%$ do sexo feminino apresentaram esse problema. Esses resultados reafirmaram o sobrepeso como o principal desvio nutricional nessa faixa etária (OMS, 2014). Já durante o estudo isso se mostrou semelhante quando avaliado a amostra como um todo, porém não se observou associação em relação à classificação nutricional, pelo IMC/idade, com os sexos (Figura 1) e (Tabela 1).

Por fim, após a ampla análise de dados, foi elaborada uma estratégia para palestra que abordasse todos os problemas evidenciados. Foi elaborado um folder explicativo com linguagem de fácil entendimento e ilustrativo que auxiliou durante a explanação das informações. Os pesquisadores, por sua vez, entregaram uma cópia do folder para cada criança, fazendo com que os interessados em mudar os hábitos de vida e alimentares se sentissem contemplados.

Inicialmente, houve resistência de parte da turma em assimilar os ensinamentos referentes a nutrição e qualidade dos alimentos. No decorrer da intervenção, após apresentação de exemplos práticos que foram facilmente associados ao cotidiano, as crianças se mostraram bastante interessadas e participativas, realizaram perguntas que envolviam a temática além de tirarem dúvidas após o término da atividade.

De modo geral, grande parte do grupo demonstrou motivação para mudarem algumas condutas rotineiras, como levar o lanche de casa e a procurar alguma atividade física fora da escola como andar de bicicleta, jogar futebol, fazer natação e dança.

\section{CONCLUSÃO}

Foi observado que mais da metade das crianças são eutróficas prevalecendo sobre elas o objetivo de prevenção da obesidade. Há uma discreta prevalência de sobrepeso e obesidade dos estudantes da escola. Para estas, o objetivo foi instruir sobre como evitar os problemas associados e a mudanças de hábitos alimentares e de vida, bem como corrigir problemas relacionados aos erros alimentares quantitativos e qualitativos. Em contrapartida notaram-se também discreta prevalência de crianças com estado nutricional abaixo do normal para idade. A intervenção foi satisfatória abrangendo o máximo de crianças possível que se demonstraram interessadas e aprenderam sobre a temática, o que sugere que as informações sobre obesidade infanto-juvenil deveriam ser sempre abordadas nas escolas, um ambiente onde os ensinamentos são levados para vida, no intuito de prevenir e/ou minimizar esse problema de saúde pública. 


\section{REFERÊNCIAS}

1. ALCÂNTARA AOR, et al. Alimentação Por Faixa Etária. Secretaria municipal adjunta de abastecimento, Belo Horizonte, 2013.

2. ALVES JGB. Atividade física em crianças: promovendo a saúde do adulto. Revista Brasileira de Saúde Materno Infantil. Recife, 2013; 3(1): 5-6.

3. AMA O, et al. Sobrepeso e obesidade infantil. Influência de fatores biológicos e ambientais em feira de Santana, BA. Sociedade Brasileira de Endocrinologia e Metabolismo, 2013; 47(2): 144-150; 2013.

4. BRASIL. Ministério da Saúde. Obesidade infantil, 2016

5. CARVALHO CA, et al. Consumo Alimentar E Adequação Nutricional Em Crianças Brasileiras: Revisão Sistemática. REVISTA PAULISTA DE PEDIATRIA, ELSEVIER, 2015.

6. CUNHA LF. A Importância de uma Alimentação Adequada na Educação Infantil. Universidade Tecnológica Federal do Paraná, Medianeira, 2014.

7. GOVERNMENT HM. Childhood Obesity: A Plan for Action. England, Crown copyright 2016

8. GROSSMAN DC, et al. US Preventive Services Task Force, Grossman DC, et al. Screening for obesity in children and adolescents: US Preventive Services Task Force recommendation statement. JAMA. 2017;317(23):2417-2426.

9. HERNANDES F, VALENTINI MP. OBESIDADE: CAUSAS E CONSEQUÊNCIAS EM CRIANÇAS E ADOLESCENTES. Revista da Faculdade de Educação Física da UNICAMP, Campinas, 2010; 8(3): 47-63.

10. KUMAR S, KELLY AS, PHD. Review of Childhood Obesity: From Epidemiology, Etiology, and Comorbidities to Clinical Assessment and Treatment. Mayo Clin Proc. 2017;92(2):251-265.

11. KUMAR S, KELLY AS. Review of childhood obesity: from epidemiology, etiology, and comorbidities to clinical assessment and treatment. Mayo Clin Proc. 2017;92(2):251-265.

12. MALTA DC, et al. Bullying em escolares brasileiros: análise da Pesquisa Nacional de Saúde do Escolar (PeNSE 2012). REV BRAS EPIDEMIOL SUPPL PeNSE 2014; 92-105

13. MARIZ LS, et al. Causas de obesidade infantojuvenil: reflexões segundo a teoria de Hannah Arendt. Texto Contexto Enferm, Florianópolis, 2015 Jul-Set; 24(3): 891-7.

14. MINISTÉRIO DO PLANEJAMENTO - Brasil. Pesquisa de Orçamentos Familiares (POF) 2008-2009: despesas, rendimentos e condições de vida. Brasília (DF): IBGE; 2010.

15. ORDWAY MR, et al. A Home Visiting Parenting Program and Child Obesity: A Randomized Trial. Pediatrics. 2018;141(2):e20171076

16. ORGANIZAÇÃO MUNDIAL DE SAÚDE. Obesidade infantil e desnutrição OMS, 2016.

17. PALLAN MJ, et al. Body image, body dissatis- faction and weight status in south asian children: a cross-sectional study. BMC Public Health. 2011;11:21-9.

18. SCHNAIDER SK, et al. Cyberbullying, school bullying, and psychological distress: a regional census of high school students. Am J Public Health. 2012;102:171-7.

19. SILVA MC, et al. Prevalência de sobrepeso e obesidade em alunos da $5^{\circ}$ a $7^{\circ}$ série do colégio Ipiranga, Belém, PA, EFDeportes.com, Revista Digital. Buenos Aires, Año 15, № 166, Marzo de 2013.

20. SOUZA EGC. Obesity, body image, and bullying in a population of schoolchildren in a town in southern Brazil.

21. RECH RR, et al. Revista da AMRIGS, Porto Alegre, 2012; 56(4): 330-334. 